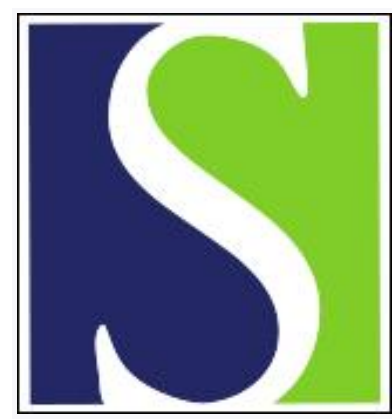

Scand J Work Environ Health 2015;41(6):594-596

https://doi.org/10.5271/sjweh.3519

Published online: 19 Aug 2015, Issue date: 01 Nov 2015

What is needed to make research on the psychosocial work environment and health more meaningful? Reflections and missed opportunities in IPD debates

by Smith PM, LaMontagne AD

We identify issues that have been overlooked in the debates about the work of the IPD-Work Consortium. These include the need for objective cut-points for psychosocial work stress exposures and better conceptual models of how psychosocial work exposures, health behaviors, obesity, and enduring health conditions are related.

Affiliation: Institute for Work \& Health, 481 University Avenue, Suite 800, Toronto ON M5G 2E9, Canada. psmith@iwh.on.ca

Refers to the following texts of the Journal: 2015;41(3):299-311 2015;41(3):312-321

The following article refers to this text: $2016 ; 42(3): 251-255$

Key terms: health; health; health behavior; IPD; IPD-Work Consortium; PAR; population attributable risk; psychosocial; psychosocial work environment; research; work stressor; working condition

This article in PubMed: www.ncbi.nlm.nih.gov/pubmed/26288154 


\section{What is needed to make research on the psychosocial work environment and health more meaningful? Reflections and missed opportunities in IPD debates}

We have read with interest the ongoing debates concerning the work of the IPD-Work Consortium focusing on the relationship between working conditions and health outcomes, which recently included a commentary from Choi and colleagues and an invited reply from Kivimäki and members of the IPD group in this journal $(1,2)$.

Our goal is not to add more fuel to this sometimes fiery debate as the commentary and reply in this journal have adequately detailed the various issues to allow readers to understand the methodological issues that should be considered when interpreting the results of the IPD-Work Consortium studies. Rather, we want to draw attention to larger issues that we feel have been overlooked to date, which impact both the work of the IPD group, but are generalizable to much of the research related to the psychosocial work environment, including research we have done ourselves. These issues concern how we measure work stressor exposures and the need for better conceptual models that recognize the complicated relationships between work stressor exposures, health behaviors, and health and other outcomes. Following this, we propose further considerations that we believe should be taken into account in the application of research in this area to workplace health promotion and disease prevention.

A large part of the debate has focused on the estimation of job strain exposure prevalence, its application in population attributable risk (PAR) estimates, and potential selection effects in the various cohorts that have been combined within the IPD-Work Consortium. While there are nuances in the way PAR have been calculated in the IPD work, which have potential impacts on the estimates, there are more fundamental issues that impact the usefulness of PAR estimates for work stressors specifically, but which also impact PAR for health behaviors and obesity. The PAR provides an estimate of the proportion of the disease that would be eliminated if an exposure were eliminated altogether $(3,4)$. The usefulness of the PAR hinges to an extent on whether the exposure of interest can be modified. For example, a smaller PAR on an exposure that has a known effective intervention is probably more useful from a population perspective than a large PAR for an exposure where public health interventions to date have had limited effectiveness. As such the usefulness of PAR for obesity, health behaviors, and job strain all hinge on whether these exposures/conditions can be successfully reduced to zero, or at least reduced substantially (3). However, the generalizability of a PAR for job strain is particularly problematic given the way job strain is estimated. The IPD work followed a long tradition of estimating job strain prevalence based on sample distributions. Given that these estimates are sample specific, it is difficult to meaningfully talk about removing or reducing job strain, as it will by default always be part of your sample even if the continuous measures are decreasing, as there is no absolute cut-point at which job strain occurs. This compromises the utility of PAR for job strain from a population health perspective.

We feel much of the debate between the Choi and Kivimäki groups would be unnecessary if there was an absolute cut-point at which exposure to job control and psychological demands was considered to be detrimental to cardiovascular risk. If this was the case, then certain samples from the IPD studies may have minimal job strain exposures (eg, if the majority of the sample was in more high-status occupations), while others may have a high prevalence job strain exposures (eg, those containing a high proportion of lower status workers). If exposure to job strain could be more consistently measured, we would not have to worry about whether sample selection and representativeness across studies resulted in a high-strain group in one sample being defined as unexposed in another. The analytical issues regarding the use of median-splits, quartiles, and other sample-specific cut-points are well documented (5), and this debate highlights the pressing need for work that helps define absolute thresholds for different psychosocial work measures and health conditions (which we note might be condition specific). These thresholds, once established, would enable PAR to be more meaningful, and studies to be more easily compared.

There is also a need for better articulation of conceptual or theoretical models to facilitate progress in understanding the complexities of the relationships between job stressor exposures, health behaviors, health, and other outcomes. Certain parts of this debate have been around what's more important as preventive targets: job stressor exposures or health behaviors. This oversimplifies the situation and creates unnecessary oppositions 
(6). A substantial body of evidence, including from the IPD Consortium, has established health behaviors as at least partial mediators of the effects of job stressors on health. While all theoretical models are provisional and subject to revision in light of evolving knowledge, we would suggest that the current state of knowledge is consistent with the model presented in figure 1 below. In this figure, the arrows between boxes represent hypothesized causal relationships, and arrows pointing to the mid-point of other arrows represent effect modification (eg, the relationship between psychosocial work exposures and distress may differ across respondents with different health behavior profiles).

Choosing between job stressor exposures and health behaviors as preventive targets also seems inappropriate for other reasons. Research to date suggests limited effectiveness of individual-directed health behavior intervention (including in the workplace), as well the potential for such strategies to exacerbate health inequalities $(7$, 8). Individual behavior change and population approaches directed at upstream determinants of health and health behaviors are complementary and have shown the most promising results to date (7). In the workplace setting, this can take the form of integrated workplace health promotion strategies targeting improvements in working conditions alongside individual health behavior change interventions. The integrated approach holds particular promise, as it has the potential for realizing preventive synergies and does not rely solely on improvements in working conditions to reduce risk or improve health. In a workplace cancer prevention study using an integrated intervention to reduce exposure to occupational carcinogens alongside workplace smoking cessation programs, double the smoking quit rate was observed in the integrated- versus standard-care control groups using a cluster randomized controlled trial design (9). Visible employer efforts to improve working conditions, likely perceived as genuine employer commitment to employee health, can serve as important motivators for individual health behavior change. Hence, even if modest associations between working conditions and health outcomes are observed, integrated work- and worker-directed intervention strategies could make a novel and valuable contribution to addressing the "wicked problems" of poor health behaviors and rising chronic disease burdens. We have recently articulated the elements of an integrated approach to work and mental health in a separate paper (10).

Further extending discussion of the implications for policy and practice of the findings of the IPD Consortium and other research in this area, there seems to be a need to take a broader view of the evidence to date. Even if job strain or other job stressors are small magnitude risk factors for health outcomes and adverse health behaviors, dismissing them as appropriate targets for preventive intervention is premature before considering the combined effects of various job stressors, both as risk factors for illness and as potentially positive influences on work performance (eg, high demand/high control "active" jobs). Job strain, for example, is related to body mass index, cardiovascular disease, common mental disorders, health behaviors, sickness absence, and other outcomes. In this regard, job stressor exposures might be considered as "fundamental causes" of work-related disease (11). With regard to implications for policy and practice, an additional consideration is the general consensus in developed countries that work should not be harmful to health and that preventable risks should be addressed. A considered integration of these two points, together with those made above, would form a better basis for the urgent task of applying this substantial body of evidence to the improvement of workplace health promotion and disease prevention strategies.

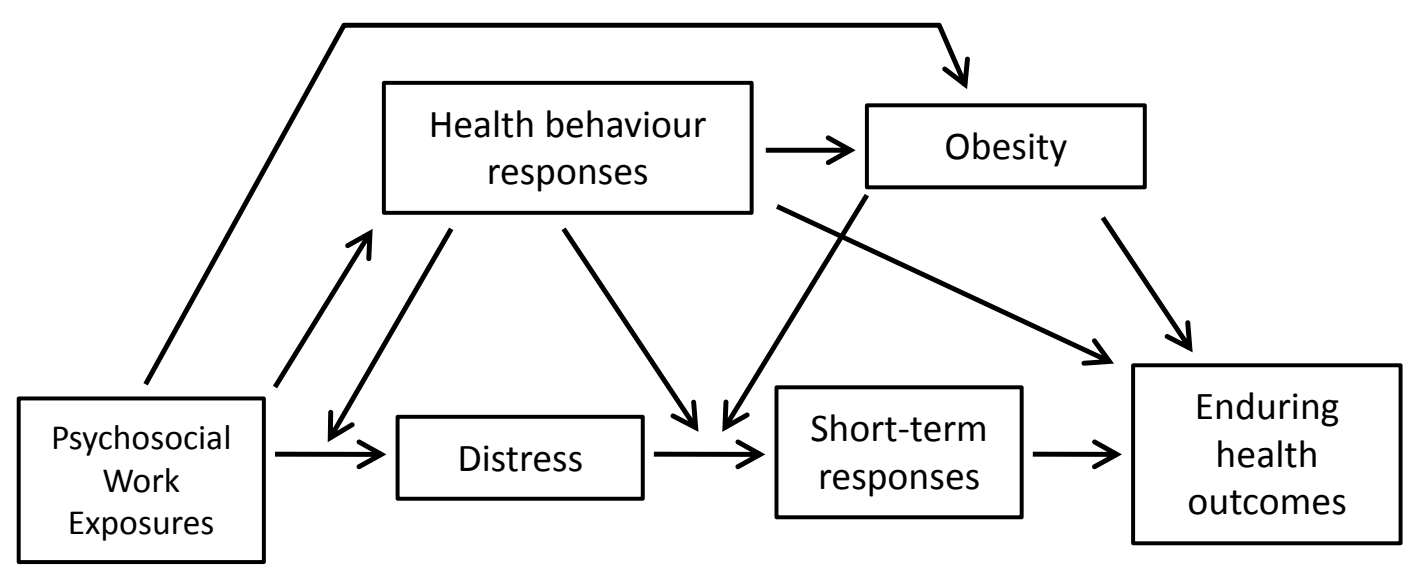

Figure 1. The complex relationships between psychosocial work exposures, health behaviors, obesity, and enduring health outcomes. Adapted from $(11,12)$. 


\section{References}

1. Choi B, Schnall P, Landsbergis P, Dobson M, Ko S, GomezOrtiz $\mathrm{V}$ et al. Recommendations for individual participant data meta-analyses on work stressors and health outcomes: comments on IPD-Work Consortium papers. Scand J Work Env Health. 2015;41(3):299-311. http://dx.doi.org/10.5271/ sjweh.3484.

2. Kivimaki M, Singh-Manoux A, Virtanen M, Ferrie JE, Batty GD, Rugulies R. IPD-Work consortium: pre-defined metaanalyses of individual-participant data strengthen evidence base for a link between psychosocial factors and health. Scand J Work Env Health. 2015;41(3):312-21. http://dx.doi. org/10.5271/sjweh.3485.

3. Greenland S. Concepts and pitfalls in measuring and interpreting attributable fractions, prevented fractions, and causation probabilities. Ann Epidemiol. 2015;25(3):155-61. http://dx.doi.org/10.1016/j.annepidem.2014.11.005.

4. Poole C. A history of the population attributable fraction and related measures. Ann Epidemiol. 2015;25(3):147-54. http:// dx.doi.org/10.1016/j.annepidem.2014.11.015.

5. Bennette C, Vickers A. Against quantiles: categorization of continuous variables in epidemiologic research, and its discontents. BMC Med Res Methodol. 2012;12:21. http:// dx.doi.org/10.1186/1471-2288-12-21.

6. Smith PM, Mustard CA. Job strain, health behaviours and heart disease. Can Med Assoc J. 2013;185(14):1251. http://dx.doi. org/10.1503/cmaj.113-2135.

7. Capewell S, Graham H. Will cardiovascular disease prevention widen health inequalities? PLoS Med 2010;7(8):e1000320. http://dx.doi.org/10.1371/journal.pmed.1000320.

8. Frohlich KL, Potvin L. The inequality paradox: The population approach and vulnerable populations. Am J Public Health 2008;98(2):216-21. http://dx.doi.org/10.2105/ AJPH.2007.114777.
9. Sorensen G, Stoddard AM, LaMontagne AD, Emmons $\mathrm{K}$, Hunt MK, Youngstrom R, et al. A comprehensive worksite cancer prevention intervention: behavior change results from a randomized controlled trial (United States). Cancer Causes Control. 2002;13(6):493-502. http://dx.doi. org/10.1023/A:1016385001695.

10. LaMontagne AD, Martin A, Page KM, Reavley N, Noblet A, Milner A et al. Workplace mental health: developing an integrated intervention approach. BMC Psychiatry. 2014;14:131. http://dx.doi.org/10.1186/1471-244X-14-131.

11. LaMontagne AD. Invited Commentary: Job strain and health behaviors - developing a bigger picture. Am J Epidemiol. 2012;176(12):1090-4. http://dx.doi.org/10.1093/aje/kws337.

12. LaMontagne AD, Keegel T, Vallance D. Protecting and promoting mental health in the workplace: Developing a systems approach to job stress. Health Promot J Austr. 2007;18(3):221-8.

Peter M Smith, PhD, 1, 2, 3 Anthony D LaMontagne, ScD 4,5

1 Institute for Work \& Health, Toronto, Ontario, Canada.

2 School of Public Health and Preventive Medicine, Monash University, Australia.

3 Dalla Lana School of Public Health, University of Toronto, Canada.

4 Population Health Strategic Research Centre, School of Health \& Social Development, Deakin University, Australia.

5 The McCaughey Vichealth Centre for Community Wellbeing, Melbourne School of Population and Global Health, the University of Melbourne, Australia.

Correspondence to: Peter M Smith, Institute for Work \& Health, 481 University Avenue, Suite 800, Toronto ON M5G 2E9, Canada. [E-mail: psmith@iwh.on.ca] 